\title{
Chirps for radar based on reciprocal time, essential discontinuities and chaotic generators
}

\author{
Horia-Nicolai TEODORESCU ${ }^{1,2}$ \\ ${ }^{*}$ Institute of Computer Science, Romanian Academy - Iasi Branch, Bd Carol I nr. 8, Iasi, Romania \\ \# "Gheorghe Asachi" Technical University, 67, Bd. D. Mangeron, 70050 Iasi, Romania
}

\begin{abstract}
We introduce and analyze a set of essentially discontinuous functions, based on multiplicative inverse of the time, which are usable as chirp signals. An advantage of these chirps is that they can be chosen from a large class to allow multiple radar operators to use each a specific waveform in automotive applications. When the new chirps are derived from chaotic signals, they are also less predictable for an observer, which is an advantage in some radar and sonar applications.
\end{abstract}

Keywords- chirp modulation; essential discontinuity; chaos; radar; autonomous vehicles; spread spectrum.

\section{INTRODUCTION}

Modern cars have several radar modules, devoted to security and driver assistance (Advanced driverassistance systems, ADAS). The tasks of these radars include collision danger detection and collision avoidance, assistance with danger detection in blind spots and rear, and assistance in drivers maneuvers such as lane change and parking. With the advent of autonomous vehicles (AVs), radar object detectors tend to clutter the roads. While strong attenuation at high frequencies $(24,77,79, \ldots, 125 \mathrm{GHz}$, see [1], [2] for details) reduce the risk of interferences at larger distances, still on busy lanes there may be hundreds of car radars at interference distances. Narrower bandwidths and specific, 'personalized' waveforms are needed to allow numerous radars interferencefree. Numerous studies have dealt with improving the waveform for automotive radars, for example [3], [4], [5], [6], [7], [8]. Frequency modulated continuous wave (FMCW) or chirp pulse radars may have advantages for this type of application.

The use of chirp signals for improving radar and sonar detection with good localization capability is well known. However, chirps based a specified, fixed modulation function are relatively easily perturbed by wide bandwidth noise and jamming. Therefore, making the modulation function more adjustable and less predictable is of interest in various fields.

In radar and sonar applications, for obtaining a precise localization (high resolution) an ideally infinitely-short pulse is needed, which is not obtainable because of too large (infinite) instantaneous power requirement for long-range detection. To alleviate this limit, a chirped pulse is emitted, and the receiving device uses a chirp-like spectrum.

The use of chaotic processes to produce chirps was established decades ago. Carroll [9] "used a chaotic map whose parameters may be optimized ..., producing different chaotic signals that ... optimized for different situations." Other authors also proposed the use of chaotic generators for radar modulators, see [10].

We introduce a set of signals that resemble chirp signals, but are obtained in a different manner, using reciprocal (multiplicative inverse) time; we name these signals 'essential discontinuity-based (pseudo-)chirps', shortly EDB chirps. The discussion is centered mostly on chirps because of easy o description, but the same principle is directly extended to FMCW radars and sonars. The proposed method may also have advantages for high resolution and small distances radars on $\mathrm{AV}$, where pulses may be as short as $1 \mathrm{~ns}$ for achieving a location precision of $3 \mathrm{~cm}$ in a $20 \mathrm{~m}$ range (see [11]).

Section 2 recalls essential discontinuities; Section 3 shows examples; the final Section is conclusive.

\section{FUNCTIONS WITH ESSENTIAL DISCONTINUITIES AND CHIRP GENERATION}

\section{A. Typical chirp impulse and FMCW signals}

Because impulse chirps and FMCW are similar in concept, except that FMCW has no break between the frequency modulated impulses (FMCW is basically a succession of concatenated chirps), we discuss them together. The FMCW typical signal, in its basic form, is described by (see equ. (1) in [12]),

$$
s(t)=A \cdot e^{j 2 \pi \frac{\Delta F}{2 T} t^{2}} \cdot e^{j\left[2 \pi\left(f_{0}+\Delta f\right) t+\varphi_{0}\right]}
$$

where $t$ is time, $\Delta F$ is the bandwidth of the modulation, $T$ is the pulse width, $f_{0}$ is the reference frequency, $\Delta f$ is the reference frequency displacement, and $\varphi_{0}$ is the signal phase. More general, the chirp signal has the form

$$
s(t)=A \cdot e^{j 2 \pi \frac{\Delta F}{2 T} \psi(t)} \cdot e^{j\left[2 \pi\left(f_{0}+\Delta f\right) t+\varphi_{0}\right]}
$$

where $\psi(t)$ is some strictly monotonic function of $t$.

\section{B. Basic concepts of function discontinuity}

We recall a few basic concepts. A real valued function $f$ defined on $\mathbf{R}$ is Hölder $\alpha$-continuous at right of some point $x_{0}$ if there is some constant $\mathrm{K}$ such that, for any pair $x_{1}, x_{2}, x_{0}<x_{1}<x_{2}$, the condition $\left|f\left(x_{1}\right)-f\left(x_{2}\right)\right|<K\left|x_{1}-x_{2}\right|^{\alpha}$. When $\alpha=$ 1 , Hölder continuity stands for standard continuity. Essential discontinuities are defined by exclusion: they are discontinuities that are not removable or ‘jump' discontinuities. We will call a Hölder essential discontinuity one that remains an essential discontinuity for whatever value of $\alpha<\infty$. Subsequently, we consider only essential 
discontinuities that are finite, that is, non-asymptotic (function not tending to infinity). For details on the discontinuity concepts, see [13], [14].

A simple example of essentially discontinuous signal is $\sin (1 / t)$. There are numerous sub-classes of (finite) essential discontinuities. For example, one can make a difference between the following classes that are introduced by the study of the sequence or function for the inverse of the variable, $1 / x$. If $f(1 / x)$ is a harmonic function, then the function $f(x)$ has a "harmonic" essential discontinuity at 0 . Similarly, zero is a point of $\alpha$-essential discontinuity at 0 if $f\left(1 / x^{\alpha}\right)$ is a harmonic function. When $f(1 / x)$ is not a harmonic function, one can say that $f(x)$ has an "anharmonic" essential discontinuity at 0 . The last category includes the cases $f(1 / x)$ toroidal, chaotic, and random.

The following properties are also useful in imagining essential discontinuities usable in chirp generation: If $f(t)$ is periodical, $f(1 / t)$ has no limit at 0 . If $f$ has derivatives up to order $n$ and if $f$ is periodical, all its derivatives are periodical. Therefore, if $f$ has derivatives up to order $n$ and if $f$ is periodical, all the derivatives of $f(1 / t)$ tend to $\infty$ at 0 .

If $f(t)$ is periodical, derivable, and has limit 0 at 0 , then $f(t) f(1 / t)$ also tends to 0 at 0 , but $f^{\prime}(t) f(1 / t)$ may have no limit at 0 . Moreover, $f(t) f^{\prime}(1 / t)$ may have no limit at 0 . Example: $f(t)=\sin t, \sin t$. $\sin \frac{1}{t} \rightarrow 0$ but $\cos t \cdot \sin \frac{1}{t}$ not $\rightarrow 0$ (has no limit).

A function is said oscillatory bounded if (i) it is bounded on $\mathbf{R}$ and (ii) it has no limit at $\infty$. For an oscillatory bounded function, the condition (ii) in the definition is equivalent with: (iii) $f(1 / x)$ has no limit at zero (has an essential discontinuity at $x=0$ ). If $f$ has derivatives, all its derivatives are unbounded at 0 in reciprocal time, $1 / t$. If $f$ is oscillatory, bounded everywhere, and has a derivative of order $n$, its derivative is oscillatory and its derivative with reciprocal time $1 / t$ is unbounded at zero.

If a system is not asymptotically stable at $\infty$ and is bounded everywhere, there is a linear function in one or several squared variables of the system such that it is oscillatory. The derivatives of that function in reciprocal time, when they exist, are unbounded and limitless in reciprocal time, at 0.

\section{Essential discontinuity-based chirps}

What we seek is to show that chirp functions, which have the general form

$$
s(t)=A \cos (a+b t+c \psi(t))
$$

where $\psi(t)$ is a (typically strictly) monotonic function in $t$ and $t \in\left[t_{k}+t_{1}, t_{k}+t_{2}\right], t_{2}>t_{1}$, have as counterpart functions with essential discontinuities at the moments $t_{k}$. In this paper we are concerned with chirp-equivalent functions

$$
s(t)=A \cdot \cos \left(a+\frac{b}{t_{k}-\left(t_{k}-\psi(t)\right)}\right)
$$

where $\lim _{\mathrm{t} \rightarrow 0} \psi(t)=0$, as well as with functions

$$
s(t)=A \cdot g\left(\frac{1}{t_{k}-\psi(t)}\right) .
$$

The simplest case of chirp based on reciprocal time, according to (3), is shown in Fig. 1, along with its spectrum. We are not aware of previous proposals of chirps based on reciprocal time.

Notice that the chirp $\sin \left(a t+b t^{2}\right)$ has a parabolic phase function $\varphi(t)=a t+b t^{2}$, with $f$ the frequency, $f=\frac{1}{2 \pi} \frac{d \varphi}{d t}=\frac{1}{2 \pi} 2 b t \quad, \quad \varphi(f)=\frac{a}{b^{\prime}} f+$ $\frac{b}{{b^{\prime}}^{2}} f^{2}, b^{\prime}=\frac{1}{\pi} b$, a parabolic function in $f$. In addition, $\varphi=c \varphi^{\prime}+d \varphi^{\prime 2}$ is the differential equation of the chirp. Instead, $\sin (1 / t)$ has decreasing phase $\varphi(f)=1 / t$ and negative instantaneous frequency $f=\frac{1}{2 \pi} \frac{d \varphi}{d t}=-\frac{1}{t^{2}}$, with a phase function $\varphi(f)=$ $\sqrt{-f}$. When using $\sin \frac{1}{t_{0}-t}$, with $\varphi(f)=\frac{1}{t_{0}-t}$, the frequency is $f=\frac{1}{2 \pi} \frac{d \varphi}{d t}=\frac{1}{\left(t_{0}-t\right)^{2}}, \varphi(f)=\sqrt{f}$.

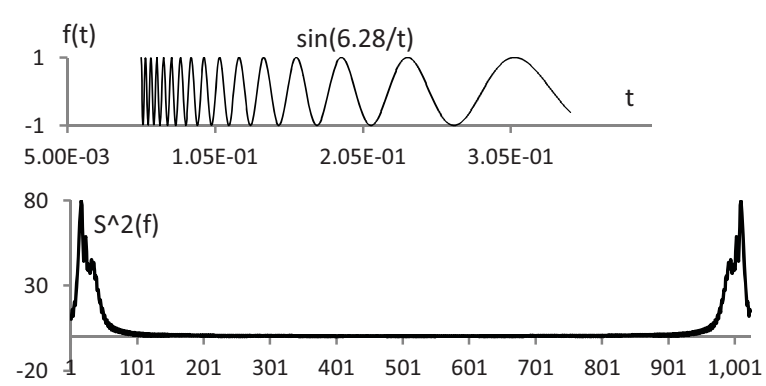

Figure 1. Signal $\sin (2 \pi / t)$ and spectrum obtained for samples from 336 up to 674, padded with 0 from 1 ro 335 and from 674 to 1024. Series $\sin (2 \pi / t)$, step of incrementing $t, \Delta t=2.74 \mathrm{E}-04$, starting from $t_{0}=5.48 \mathrm{E}-02$ (for sample \#336). No window applied.

We name chaotic essential discontinuity at $x_{0}$ any essential discontinuity of a function $g$ continuous on a left-closed interval (or right-closed) and is produced by a chaotic process $f(t)$ as $g(x)=f(1 / t)$.

\section{CHAOTIC SIGNALS WITH DISCONTINUITIES}

\section{A. Introductory issues}

Let $x(n)$ be a chaotic signal, e.g., produced by one of Sprott's circuits [15], or circuits as in [16], [17-18], or a logistic series etc. Define

$$
\begin{gathered}
y(u)= \\
=\left\{\begin{array}{c}
x(n) \quad \text { for } \frac{1}{u}=n \in N \\
\left(x\left(\left\lfloor\frac{1}{u}\right\rfloor+1\right)-x\left(\left\lfloor\frac{1}{u}\right\rfloor\right)\right) \cdot\left(u-\left\lfloor\frac{1}{u}\right\rfloor\right) \text { for }\left\lfloor\frac{1}{u}\right\rfloor \notin N
\end{array}\right.
\end{gathered}
$$

Then, there is no $\lim _{u \rightarrow 0} y(u)$. Also, there is no $\lim _{u \rightarrow 0} g(y(u))$, where $g$ is some function, e.g., $\sin ()$.

\section{B. Examples}

Recall that the logistic map is $x_{n+1}=a x_{n}$. $\left(1-x_{n}\right)$; also chaotic is $x_{n+1}=a x_{n}^{p} \cdot\left(1-x_{n}^{p}\right)$, $p \in \boldsymbol{N}$ and suitable $a$. Fig. 2 shows a chirp based on the logistic map in reciprocal time. Figs. 3 and 4 show chirps based on the logistic map and functions of the reciprocal time, based on (5). Fig. 5 provides another example together with the spectrum of the chirp. Notice that the graphs are drawn using linear interpolation between the generated points; therefore, the signal equation should be written for all intervals 
$\left[\frac{1}{n+1}, \frac{1}{n}\right]$ as $x(t)=x(n+1)+\frac{x(n)-x(n+1)}{\frac{1}{n+1}-\frac{1}{n}}\left(t-\frac{1}{n+1}\right)$. To derive the Fourier spectrum, the sampling of the above should be done with a maximal sampling period of $T_{\text {sampl }} \ll 1 / N(N-1)$, where $N$ is the maximal value of the index in the series $\left\{x_{n}\right\}_{n=0, \ldots, N}$.
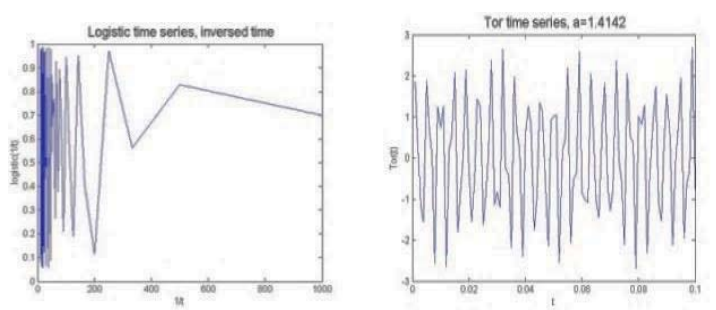

Figure 2. Generating an essentially discontinuous chaotic chirp signal with the logistic map, with a toroidal regime.

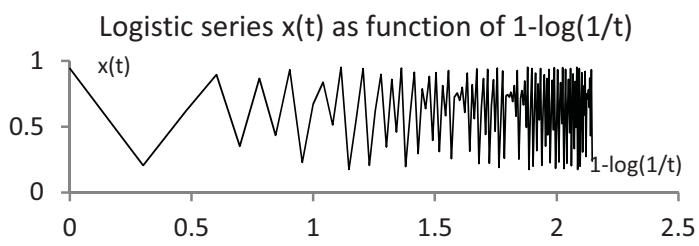

Figure 3. Pseudo chirp obtained using the logistic map as a fucntion of $1-\log (1 / t)$.

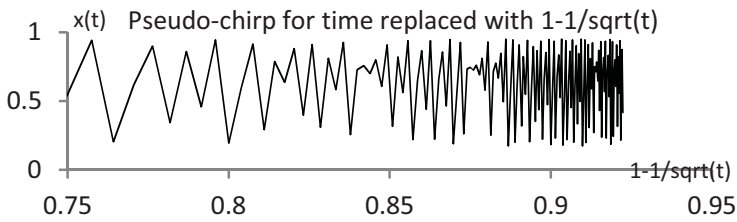

Figure 4. Pseudo chirp obtained using the logistic process as a fucntion of $1-\log (1 / \sqrt{t})$.
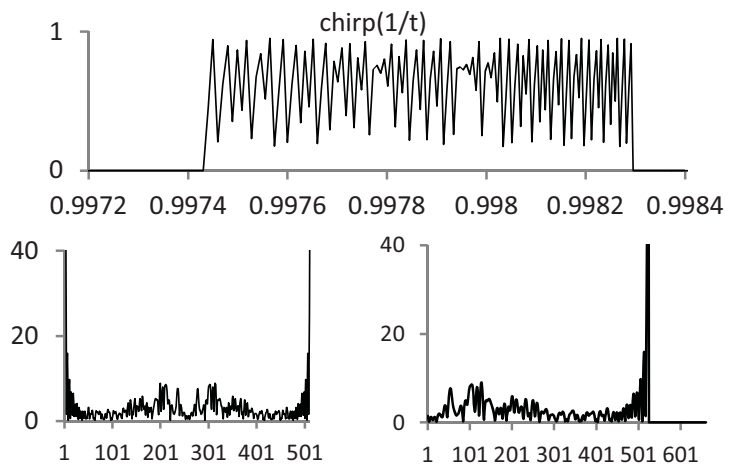

Figure 5. Logistic chirp in reciprocal time and spectrum (spectrum truncated at amplitude 40 for better perceptibility.) Cases of periods of 512 and 1024 samples, chirp of 128 samples.

Notes: The use of reciprocal time requires supplementary technical means to implement the operation. One way is to record in a LIFO memory the samples generated by a chaotic system and then produce them as radar signal at the corresponding reciprocal time moments. If the reciprocal time is between $\left[\frac{1}{t_{1}}, \frac{1}{t_{N}}\right], \frac{1}{t_{N}}>\frac{1}{t_{1}}$, then the smallest time interval between samples is $t_{N}-t_{N-1}=\delta t$. The value of $\delta t$ is the sampling period for the original signal. The reciprocal time values are then $1 / t_{N-k}=$ $1 /\left(t_{N}+k \delta t\right)$.

\section{Further theoretical issues}

The class of functions that can be used to produce EDB chirps is very large (infinite). The next properties help detailing this statement. In general, when $x(t)$ is bounded but has no limit at infinity, $x\left(1 / t^{\alpha}\right), \alpha>1$ has an essentially discontinuity at (no limit at) zero. In addition, if $P_{n}(t)$ is a polynomial and $x_{i}$ are its real roots, $x\left(1 / P_{n}(t)\right)$ is essentially discontinuous at $x_{i}$.

Property. There is an injective mapping from the set of trajectories starting from different initial values of a chaotic time series and the set of sequences with essentially no limit. Also, there is an injective mapping from the set of trajectories starting from different initial values of chaotic functions with specified initial condition $f\left(t \in[1, \infty), x_{0}\right)$ and the set of functions with essential discontinuity (essentially no limit) at 0 .

By fixing arbitrarily the initial condition, one obtains the following property:

Property. There is an injective mapping from the set of chaotic functions $f$ :Time $\rightarrow \boldsymbol{R}^{n}$ with specified initial condition $f\left(t \in[1, \infty), x_{0}\right)$ and the set of functions with essential discontinuity (essentially no limit) at 0 . Thus, the study of chaotic time series could be brought to the study of a subset of bounded sequences $x_{1 / n}$ with no limit for $1 / n \rightarrow 0$ (trivial).

Remark. Any bounded continuous function $f(t)$ with no asymptotic limit at infinity (divergence at infinity) has the property that it is divergent at zero for reversed time, $f(1 / t)$ (essential discontinuity at zero).

All harmonic functions $g \in H$ and thus all finite order trigonometric polynomials with at least one nonnull coefficient) are oscillating at infinity and therefore have no limit at zero in reciprocal time; similarly for all chaotic functions. Notice that the density of points $f(1 / t)=0$ in the vicinity of zero, defined as $\frac{\log n([\varepsilon, \varepsilon+\Delta \varepsilon])}{\log \Delta \varepsilon} \sim \varepsilon$

If $f$ is periodical with period $T$, then there are $\left\lfloor\frac{1}{T}\left(\frac{1}{\varepsilon}-\frac{1}{\varepsilon+\Delta \varepsilon}\right)\right\rfloor=\left\lfloor\frac{1}{T} \cdot \frac{\Delta \varepsilon}{\varepsilon \cdot(\varepsilon+\Delta \varepsilon)}\right\rfloor$ such points in $[\varepsilon, \varepsilon+$ $\Delta \varepsilon]$. For $\Delta \varepsilon=\varepsilon$, one obtains $\left\lfloor\frac{1}{T} \cdot \frac{1}{2 \varepsilon}\right\rfloor$, which tends to infinity for $\varepsilon \rightarrow 0$, with the density $\rho(\varepsilon)=$ $\frac{n([\varepsilon, \varepsilon+\Delta \varepsilon])}{\varepsilon}=\frac{1}{\varepsilon} \cdot\left\lfloor\frac{1}{T} \cdot \frac{1}{2 \varepsilon}\right\rfloor$ tending quadratically in $\frac{1}{\varepsilon}$ to infinity. So, any process for which there is no constant value A such that $\rho(\varepsilon)=\frac{A}{\varepsilon^{2}}$ is not periodical; it may be slower than periodical when the time is stretched, like for $\sin \left(a t+b t^{2}\right)$, or it can be faster than periodical when time is compressed, such as for $\sin (\sqrt{t})$ or for $\sin (\ln (t))$.

Assume that $f(t) \in[-1,1]$. The constant $T$ that minimizes for a chaotic process $f(t)$ the expression

$$
T=\arg \min _{\tau} \int_{-\infty}^{\infty}|1-\arcsin (f(t / \tau))| d t
$$

if the integral and the minimum exist is named the pseudo-period of the chaotic process. The waveform used in the chirp according to the method described in this article should include more than a single pseudoperiod, for reasons of robustness of the detection. 
Let $y=\sin \left(\frac{1}{t}\right)$. Then, $y^{\prime}=-\frac{1}{t^{2}} \cos \left(\frac{1}{t}\right), y^{\prime \prime}=$ $\frac{1}{t^{4}} \sin \left(\frac{1}{t}\right)+\frac{2}{t^{3}} \cos \left(\frac{1}{t}\right)$, or $y^{\prime \prime}=\frac{1}{t^{4}} y-\frac{2}{t} y^{\prime}$. Similar equations can be found that are satisfied by $y=$ $\cos (1 / t)$ and other functions.

Consider $g(x)$ of bounded variation for $[1, \infty)$, with no zero (fixed point) and satisfying $\lim _{\mathrm{x} \rightarrow \infty} g(x)=$ $c$. Let $f$ be of unbounded variation on any interval $[a, \infty)$. Then, $g(f(1 / x))$ has unbounded variation at 0 .

In principle, chaotic chirps have a spectrum unpredictable by observers (anti-active jamming of the radars). In this case, the radar knows what the correct chirp spectrum is and thus the correct chirpdecoding filters to use in the next chirp bursts, while the attacker does not. The next chirp may be generated by the same chaotic process or by one with modified parameters.

\section{DISCUSSION AND CONCLUSIONS}

The typical chirp signals in the literature are continuous on $\mathbf{R}$, with no limit at infinity. We proposed the use of chirps that (i) have essential discontinuities at the specified time moments $\left\{t_{k}\right\}_{k=1 \ldots \infty}$; (ii) where $t_{k}$ are generated pseudorandomly or chaotically, and (iii) the signals generated at each time moment are functions with essential discontinuities, moreover (iv) the functions are produced as solutions (realizations) of chaotic processes with specified initial conditions. In specific applications, (i)) can be replaced by uniformly distributed time moments.

The wide spectrum of these essential discontinuitybased pseudo-chirps may help improving the detection of multiple targets (resolution) and may be more resistant to jamming.

Carroll [9] suggests that "chaos-based signals may have advantage in the detection of complex targets". The same author "found that one can optimize a chaos-based signal ...thus allowing the identification of a complex target." We believe that the same advantage may occur for the proposed signals, with the extra advantage of better optimization capabilities.

It is unclear for now what benefits can be obtained using EDB chirps under interferences [19] and under clutter conditions as in [20], but following [9] we believe that benefits are achievable with EDB chirps.

Finally, the use of these shorter chirps may help reduce the radiation dose (Specific absorption rate, SAR) for pedestrians exposed to automotive radars. While radars operate in the range $20 \mathrm{GHz}-85 \mathrm{GHz}$, they produce non-ionizing radiation and their heating effect is limited by standards such as ICNIRP. At the levels for automotive radars, radars are not dangerous, but their field adds up to other radiation sources.

Note. This study was not commissioned or supported by the institutions to which I am affiliated. It has no relation to my work at those institutions and was fully financially supported by the author.

\section{REFERENCES}

[1] R. Mende, H. Rohling, New automotive applications for smart radar systems smart microwave sensors. Report, 2002, Smartmicrogroup.com, www.smartmicrogroup.com/ fileadmin/user upload/Documents/Publications/New Autom otive_Applications_for_Smart_Radar_Systems_V5.p̄pdf.

[2] J. Wenger, "Automotive radar - status and perspectives," IEEE Compound Semiconductor Integrated Circuit Symposium, 2005. CSIC '05., Palm Springs, CA, USA, 2005, pp. 4 pp.-.doi: 10.1109/CSICS.2005.1531741.

[3] H. Rohling, C. Moller, "Radar waveform for automotive radar systems and applications," IEEE Radar Conf., Rome, 2008, pp. 1-4. doi: 10.1109/RADAR.2008.4721121.

[4] H. Rohling, M.-M. Meinecke, "Waveform Design Principles for Automotive Radar Systems". Proc. Int. Conference Radar, 2001 CIE, pp.1-4. 10.1109/ICR.2001.984612.

[5] D.A. Williams, "Millimetre wave radars for automotive applications," 1992 IEEE MTT-S Microwave Symposium Digest, Albuquerque, NM, USA, 1992, pp. 721-724 vol. 2. doi: 10.1109/MWSYM.1992.188086.

[6] L. Roselli et al., "A cost driven $24 \mathrm{GHz}$ Doppler radar sensor development for automotive applications," European Radar Conference, 2005. EURAD 2005, Paris, 2005, pp. 335-338. doi: 10.1109/EURAD.2005.1605632.

[7] J. Schoebel et al., "Design considerations and technology assessment of phased-array antenna systems with RF MEMS for automotive radar applications," IEEE Trans. Microwave Theory and Techn., vol. 53, no. 6, pp. 1968-1975, June 2005.

[8] M. Skutek, M. Mekhaiel and G. Wanielik, "A precrash system based on radar for automotive applications," Proc. IEEE IV2003 Intelligent Vehicles Symp., Columbus, OH, USA, 2003, pp. 37-41. doi: 10.1109/IVS.2003.1212879.

[9] T.L. Carroll, Optimizing chaos-based signals for complex radar targets, Chaos 17, 033103 (2007); doi.org/10.1063/1.2751392.

[10] N.K. Alapati, R.H. Kirklin, P.C. Etter, "Analysis of chaotic waveforms for application to active sonar systems." Final Report. SA Radix Systems Inc. Gaithersburg, MD 20877, Jun 1993, https://apps.dtic.mil/dtic/tr/fulltext/u2/a266368.pdf.

[11] M. Klotz, H. Rohling, "A 24 GHz short range radar network for automotive applications," 2001 CIE Int. Conf. on Radar Proc., Beijing, China, 2001, pp. 115-119. doi: 10.1109/ICR.2001.984635.

[12] M. Steinhauer, H. Ruob, H. Irion, W. Menzel, "Millimeterwave-radar sensor based on a transceiver array for automotive applications," IEEE Trans. Microwave Theory and Techniques, vol. 56, no. 2, pp. 261-269, Feb. 2008.

[13] J Klippert (2000), "On a discontinuity of a derivative". Int. J. Mathematical Education in Science and Technology, 31:S2, pp. 282-287, DOI:10.1080/00207390050032252.

[14] O. John, J. Malý, J. Stará, "Nowhere continuous solutions to elliptic systems." Commentationes Mathematicae Universitatis Carolinae, Vol. 30 (1989), No. 1, 33-43.

[15] Sprott, JC, “A new class of chaotic circuit." Physics Letters A, Vol. 266, no. 1, pp. 19-23, Feb 142000.

[16] M. Stork, J. Hrusak, D. Mayer, "A new chaotic system based on state space energy feedback." Int. Conf. Appl. Electronics, Pilsen, Czech Republic, Sep 8-9, 2015, pp. 229-232.

[17] H.N. Teodorescu, "Simple nonlinear circuits and applications to chaotic sensors and chaos generators." Int. Conference on Applied Electronics (AE), Book Series: Applied Electronics, Pilsen, Czech Republic, Sep 05-06, 2017, pp. 243-246.

[18] H.N. Teodorescu; V. Cojocaru, "Complex signal generators based on capacitors and on piezoelectric loads. In: Chaos Theory: Modeling, Simulation and Applications, C.H. Skiadas, Dimotikalis, I., C. Skiadas (Eds.), pp. 423-430. 2011.

[19] A. Bultan, A.N. Akansu, "A novel time-frequency exciser in spread spectrum communications for chirp-like interference," Proc. 1998 IEEE Int. Conf. Acoustics, Speech and Signal Proces., ICASSP '98, Seattle, WA, USA, 1998, pp. 3265-3268 vol. 6. doi: 10.1109/ICASSP.1998.679561.

[20] F. Gini, M. Montanari, L. Verrazzani, "Estimation of chirp radar signals in compound-gaussian clutter: A cyclostationary approach.” IEEE Trans. Signal Processing, Vol. 48, No. 4, April 2000, pp. 1029-1039. 